\title{
Biomimetics and cilia propulsion
}

\author{
MARTIN R. MAXEY
}

Division of Applied Mathematics, Brown University, Providence, RI 02912, USA

Many swimming microorganisms are able to propel themselves by the organized beating motion of numerous short flagella or cilia attached to their body surface. For their small size and the inherently viscous nature of the motion, this mechanism is very effective and they can swim several body lengths per second. The quest has been to see if artificial cilia may be developed and if the strategy of cilia propulsion can be used in microfluidic devices to transport fluids in a localized and controllable manner. Babataheri et al. (J. Fluid Mech., this issue, vol. 678, 2011, pp. 5-13) explore the response of chains of small paramagnetic beads that are elastically bonded together to form artificial cilia. The chain or fleximag is tethered to the surface and driven by external magnetic fields, responding also to both fluid and elastic forces. A key observation from their experiments and model is that for a simple planar-forcing strategy there is a hidden symmetry that limits the net transport of fluid.

Key words: low-Reynolds number flows, MEMS/NEMS, swimming/flying

\section{Introduction}

As described by Purcell (1977), microorganisms - be they bacteria, spermatozoa or unicellular organisms such as Paramecium - must rely on specialized swimming strategies to propel themselves in a viscous medium. Any reversible motion of appendages will simply move the organism in one direction and then back again with no net forward motion. This reversibility of Stokes flow is demonstrated in the famous experiments by Taylor (1967) showing the stretching of a dye spot in a Couette flow and the motion of self-propelled bodies. A bacterium such as Caulobacter crescentus swims at speeds of $40 \mu \mathrm{m} \mathrm{s}^{-1}$ propelled by a single flagellum that rotates several hundred times a second, driven by a molecular motor embedded in the cell membrane. The flagellum, a loose spiral filament about $20 \mathrm{~nm}$ in diameter, produces a corkscrew motion of the flagellum that overcomes the reversibility constraint. The term 'corkscrew' is illustrative but the key is the asymmetry of viscous drag forces on a cylinder. In a Stokes flow, a long cylinder will experience up to twice the viscous resistance to motion transverse to the axis than parallel to it. The helical form of the flagellum presents less tangential drag than normal resistance to the filament. So as long the flagellum turns the rotational torque of the motor is converted to a net force along the axis of the helix (Li \& Tang 2006).

Cilia, which may be $10 \mu \mathrm{m}$ long, and the flagella of spermatozoa, which may be $40-80 \mu \mathrm{m}$ in length, with diameters of $200 \mathrm{~nm}$ have a more complex structure. Both consist of a bundle of cross-linked filaments that actively flex along the whole length (Brennen \& Winet 1977). So rather than passively responding to a motor, the beating 

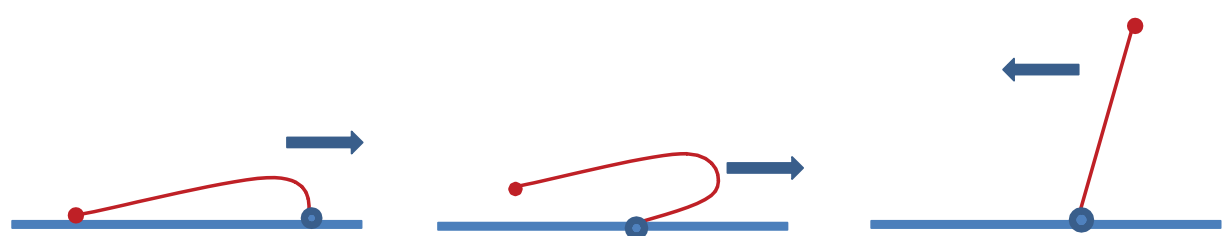

FIGURE 1. Sketch of a natural cilium stroke: initial stage of return stroke, mid-return stroke and power stroke with faster sweep from right to left; cilium remains close to surface during return. Markers are shown for the fixed base of the cilium and for the tip.

motion of a flagellum or a cilium is the result of active forcing and the relative sliding of the filaments. The flagellum of a spermatozoon generates a travelling wave along its length in the planar beating motion creating the essential asymmetry for propulsion (Lauga \& Powers 2009).

Larger microorganisms such as Paramecia are several hundred microns in size and are propelled several body lengths per second by the beating of cilia which cover their surface. Cilia, coating the airways of the lungs, drive the transport of mucus and clear debris to keep the airways open (Sleigh, Blake \& Liron 1988). The keys to natural propulsion by cilia are the motion of an individual cilium and their cooperative beating within an array of closely spaced cilia. Figure 1 shows an outline of a typical motion for a cilium. There is a rapid power stroke where the cilium is nearly straight and sweeps in a transverse motion. In the slower recovery motion the cilium is folded and returns closer to the surface presenting a more tangential motion relative to the surrounding fluid. The return stroke may also involve a sideways sweeping arc (Brennen \& Winet 1977). The net result is a larger net force and transport of the fluid for the power stroke than from the return motion. The challenge is to see if we can harness these mechanisms for the effective pumping of fluids in microdevices using artificial cilia.

\section{Overview}

The fleximag artificial swimmer is among the most intriguing of recent biomimetic devices. First demonstrated by Dreyfus et al. (2005), the flagellum of the swimmer consists of a chain of micron-scale, super-paramagnetic beads elastically bonded together. This flagellum or fleximag is attached to a larger non-magnetic particle, a red blood cell in the original experiments. Super-paramagnetic beads acquire a magnetic dipole moment when placed in a uniform magnetic field and if they are in a random suspension they will spontaneously self-assemble into chains aligned with the field. If the field strength oscillates or rotates, the chain will turn to align with the changing field direction but its motion is limited by viscous drag forces. Any misalignment produces a reduction in the magnetic attraction between adjacent beads and even a repulsion force for larger misalignments. The overall effect, if the beads are elastically bonded, is for the chain to flex and bend. In the experiments, a planar oscillation of the magnetic field about a primary direction produces a planar beating motion of the fleximag. The phase of the transverse deflections appears as a travelling wave from the free end of the chain towards the body (Dreyfus et al. 2005). 
The swimmer moves tail first, opposite to the usual motion of a spermatozoon but consistent with the reversed direction of the travelling wave. A field that rotates in a cone about an axis is equally effective in producing flexing and propulsion (Keaveny \& Maxey 2008).

Babataheri et al. (2011) take this concept one stage further and create an artificial cilium by attaching a fleximag to a rigid surface and applying an oscillating magnetic field. They demonstrate a range of responses driven in turn by a planar oscillation about a primary field normal to the wall, a three-dimensional rotating field and asymmetric planar oscillations. In each case the fleximag deforms in response to the combination of magnetic forces, viscous fluid forces and elastic forces. The fluid forces are further influenced by the presence of the rigid surface. The ratios of these different forces are characterized by a set of non-dimensional parameters. The Mason number, $M a$, characterizes the ratio of the viscous forces to magnetic forces and the magnetoelastic number, $M n$, characterizes the ratio of magnetic forces to elastic forces in the chain. A third parameter is the sperm number, $S p$, that is linked to the ratio of viscous forces to elastic forces as

$$
S p^{4} \propto M a \times M n .
$$

$S p$ scales linearly with the length $L$ of the fleximag chain.

We comment here to highlight a few points. The Mason number, in the present context, is proportional to the frequency of oscillation of the magnetic field. With slow oscillations, $M a \ll 1$, viscous forces do not significantly slow the rotation of the chain and it remains straight, aligned with the field at all times. Thus, a slow planar sweep of the magnetic field and the fleximag would be ideal for the power stroke of a cilium, illustrated in figure 1. The usual power stroke of a natural cilium is fast, but the key issue is the total displacement of the stroke and this is the same whether the sweep is slow or fast.

The challenge is to develop an effective recovery stroke. Here deformation of the fleximag is desirable and so a faster return stroke with larger $M a$ is the option to consider. Experience from studies of artificial swimmers can shed light on this. The experiments (Dreyfus et al. 2005) covered the range $M n=4-16$ and optimal swimming speeds were achieved with $S p=2-4$. Under these conditions, the chain bends along the full length. At low $S p$, the chain oscillates rigidly as a straight rod in a reversible motion, while at large $S p$, only a section at the free end is deflected and the rest of the chain does not flex.

A significant contribution of the paper (Babataheri et al. 2011) is to test experimentally some of the cited theoretical results and determine scaling relations for the response. The first step is to consider simple oscillations or rotation of the magnetic field and determine both the amplitude and waveform of the fleximag as a function of the frequency, or $M a$. In their figure 2, they show that over a broad range of conditions the amplitude of the free end motion, relative to the length of the chain $L$, scales as $M a^{-1 / 2}$. The authors focus on the effects of strong magnetic fields, where $M n \sim 10^{5}$, and elastic bending moments play a secondary role.

Importantly, the authors demonstrate that even with an asymmetric forcing, and what may appear to be a winning strategy for the return stroke of the artificial cilium, the net force exerted on the fluid is quite small. Underlying the present simple strategy, at first approximation, is a correlation between the force exerted on the fluid and the torque at the base of the chain. Since the latter is necessarily zero for any reciprocal motion, the total net force is limited. 


\section{Future}

The quest for an effective artificial cilium continues. Several strategies are possible based on the fleximag concept and these remain to be explored. One is to use larger amplitude oscillations in the direction of the magnetic field whereby a stronger flexing motion and a nonlinear response is induced (Coq et al. 2010). This may lead to a recovery stroke closer to that of a natural cilium. Another is to use a magnetic field that rotates in a tilted cone. (Compare this with figure $2 c$ of Babataheri et al. 2011.) Vilfan et al. (2010) have demonstrated a pumping action based on this strategy using non-bonded magnetic chains. While described as artificial cilia, it is closer in concept to attaching bacteria to a surface in a carpet as in the experiments by Darnton et al. (2004). The magnetic field rotating about the inclined axis causes the chains to precess in a manner similar to the rotation of the flagellum of a bacterium. A fleximag permits larger amplitude rotations. Many other questions and options remain to be tested. Going beyond the response of a single cilium is how cilia respond collectively. Natural cilia are clustered in a carpet and a phase-locking mechanism is observed; the cilia beating appears as a travelling wave that sweeps across the carpet. The source of these metachronal waves, which are energetically favourable, is believed to be hydrodynamic and not the result of some neural signalling (Gueron \& Levit-Gurevich 1999). While the present quest for effective artificial cilia may be seen as an engineering issue, the questions posed provide important insights into just how successful natural cilia are in their function.

\section{References}

Babataheri, A., Roper, M., Fermigier, M. \& Roure, O. D. 2011 Tethered fleximags as artificial cilia. J. Fluid Mech. 678, 5-13.

Brennen, C. \& Winet, H. 1977 Fluid mechanics of propulsion by cilia and flagella. Annu. Rev. Fluid Mech. 9, 339-398.

Coq, N., Roure, O. D., Fermigier, M. \& Bartolo, D. 2010 Three-dimensional beating of magnetic microrods. Phys. Rev. E 82, 041503.

Darnton, N., Turner, L., Breuer, K. \& Berg, H. C. 2004 Moving fluid with bacterial carpets. Biophys. J. 86, 1863-1870.

Dreyfus, R., Baudry, J., Roper, M. L., Fermigier, M., Stone, H. A. \& Bibette, J. 2005 Microscopic artificial swimmers. Nature 437, 862-865.

Gueron, S. \& Levit-Gurevich, K. 1999 Energetic considerations of ciliary beating and the advantage of metachronal coordination. Proc. Natl Acad. Sci. USA 96, 12 240-12245.

Keaveny, E. \& Maxey, M. 2008 Spiral swimming of an artificial micro-swimmer. J. Fluid Mech. 598, 293-319.

Lauga, E. \& Powers, T. 2009 The hydrodynamics of swimming microorganisms. Rep. Prog. Phys. 72, 096601.

LI, G. \& TANG, J. 2006 Low flagellar motor torque and high swimming efficiency of Caulobacter crescentus swarmer cells. Biophys. J. 91, 2726-2734.

Purcell, E. M. 1977 Life at low Reynolds number. Am. J. Phys. 45, 3-11.

Sleigh, M., Blake, J. \& Liron, N. 1988 The propulsion of mucus by cilia. Am. Rev. Respir. Dis. 137, 726-741.

TAYLOR, G. 1967 Low Reynolds number flow. In U.S. National Committee for Fluid Mechanics. Educational Development Center Inc.

Vilfan, M., Potocnik, A., Kavcic, B., Osterman, N., Vilfan, A. \& Babic, D. 2010 Self-assembled artificial cilia. Proc. Natl Acad. Sci. USA 107, 1844-1847. 